\title{
Emergence of a Magnetostructural Dipolar Glass in the Quadruple Perovskite Dy1- $\delta \mathrm{Mn} 7+\delta 012$
}

\author{
R. D. Johnson, D. D. Khalyavin, P. Manuel, L. Zhang, K. Yamaura, \\ and A. A. Belik
}

\section{Published version information}

Citation: R Johnson et al. "Emergence of a Magnetostructural Dipolar Glass in the

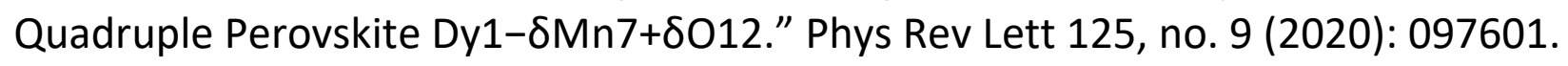

DOI: $10.1103 /$ PhysRevLett.125.097601

This version is made available in accordance with publisher policies. Please cite only the published version using the reference above. This is the citation assigned by the publisher at the time of issuing the APV. Please check the publisher's website for any updates. 


\title{
Emergence of a Magnetostructural Dipolar Glass in the Quadruple Perovskite $\mathrm{Dy}_{1-\delta} \mathbf{M n}_{7+\delta} \mathbf{O}_{12}$
}

\author{
R. D. Johnson, ${ }^{1, *}$ D. D. Khalyavin, ${ }^{2}$ P. Manuelø, ${ }^{2}$ L. Zhang, ${ }^{3,4}$ K. Yamaura $\odot,{ }^{3,4}$ and A. A. Belik ${ }^{3}$ \\ ${ }^{1}$ Department of Physics and Astronomy, University College London, Gower Street, London WC1E 6BT, United Kingdom \\ ${ }^{2}$ ISIS facility, Rutherford Appleton Laboratory-STFC, Chilton, Didcot OX11 OQX, United Kingdom \\ ${ }^{3}$ International Center for Materials Nanoarchitectonics (WPI-MANA), National Institute for Materials Science (NIMS), \\ Namiki 1-1, Tsukuba, Ibaraki 305-0044, Japan \\ ${ }^{4}$ Graduate School of Chemical Sciences and Engineering, Hokkaido University, \\ North 10 West 8, Kita-ku, Sapporo, Hokkaido 060-0810, Japan
}

(Received 6 April 2020; revised 15 May 2020; accepted 28 July 2020; published 26 August 2020)

\begin{abstract}
We show that a polar, pseudo-Jahn-Teller instability exists for the underbonded rare-earth $A$-site cations in the quadruple perovskite $\mathrm{Dy}_{1-\delta} \mathrm{Mn}_{7+\delta} \mathrm{O}_{12}$, which leads to the spontaneous formation of a dipolar glass. This observation alone expands the applicability of pseudo-Jahn-Teller physics in perovskite-derived materials, for which it is typically confined to $B$-site cations. We demonstrate that the dipolar glass order parameter is coupled to a ferrimagnetic order parameter via strain, leading to a first order magnetostructural phase transition that can be tuned by magnetic field. This phenomenology may emerge in a broad range of perovskite-derived materials in which $A$-site cation ordering and octahedral tilting are mutually tied to meet the criteria of structural stability.
\end{abstract}

DOI: 10.1103/PhysRevLett.125.097601

The spontaneous formation of electric dipoles in dielectrics can lead to the emergence of ferroelectricity [1], relaxor ferroelectricity [2], or antiferroelectricity [3], and the fundamental study of these properties has generated considerable impact through their application in solid-state technology. This impact might be expanded if one can deterministically couple the electric dipolar order with magnetic order in materials known as multiferroics [4]. Arguably, the most prominant candidate materials in this line of research are transition metal oxides with a perovskite-derived crystal structure (general chemical formula $A B \mathrm{O}_{3}$ ). Oxygen anions form a framework of corner-shared octahedra that coordinate $B$-site cations, while the $A$-site cations occupy the framework voids. The formation of electric dipoles in the $\mathrm{ABO}_{3}$ system is typically achieved by either (a) incorporating a $d^{0}$ transition metal ion on the $B$ sites (e.g., $\mathrm{Ti}^{4+}$ ) that supports polar, quasi-Jahn-Teller distortions $[5,6]$, (b) incorporating lone pair electrons on the $A$ sites (e.g., $\mathrm{Pb}^{2+}$ or $\mathrm{Bi}^{3+}$ ) [7-9], or (c) special combinations of octahedral rotations and cation ordering in so-called hybrid-improper ferroelectrics [10]. In principle, magnetism can be incorporated into all these scenarios.

Here, we consider an alternative approach to establish electric dipole instabilities in perovskite-derived materials; we aim to exploit quasi-Jahn-Teller displacements of underbonded $A$-site cations instead of $B$-site cations. The bonding requirements of $A$-site cations are usually well accommodated by the inherent structural flexibility of octahedral tilts and rotations [11], for example, the socalled $\mathrm{GdFeO}_{3}$ structural distortion [12] of the rare-earth simple perovskite manganites, $R \mathrm{MnO}_{3}(R=$ rare earth, yttrium, or bismuth). However, in the $\left(R \mathrm{Mn}_{3}\right) \mathrm{Mn}_{4} \mathrm{O}_{12}$ family of quadruple perovskite manganites the octahedral tilts are defined by a $1: 3$ ordering of $R$ and $\mathrm{Mn} A$-site cations, respectively [13]. In this case the octahedral tilts primarily establish a square planar coordination for the majority Mn sites, which imposes considerable rigidity in the bonding of the minority $R$ cations. It is feasible, therefore, that $R$ cations with small ionic radii will be underbonded in this quadruple perovskite framework, and hence create a local dipolar instability through the quasiJahn-Teller effect [5].

In this Letter, we demonstrate that an $A$-site dipolar instability exists in $\mathrm{Dy}_{1-\delta} \mathrm{Mn}_{7+\delta} \mathrm{O}_{12}$, which is characterized by an isostructural monoclinic strain, negative thermal expansion, and off-centering displacements of Dy that form a dipolar glass. Furthermore, we show that this structural instability is coupled to a ferrimagnetic order parameter via the strain, giving rise to a strongly first order magnetostructural phase transition that can be tuned by an applied magnetic field.

A sample with nominal chemical composition $\left(\mathrm{Dy}_{0.911} \mathrm{Mn}_{0.089}\right) \mathrm{Mn}_{7} \mathrm{O}_{12}$ (hereafter referred to as $\mathrm{DMO}$ ) was prepared from a stoichiometric mixture of $\mathrm{Mn}_{2} \mathrm{O}_{3}$ and $\mathrm{Dy}_{2} \mathrm{O}_{3}$ (99.99\% purity). Single-phase $\mathrm{Mn}_{2} \mathrm{O}_{3}$ was prepared from commercial $\mathrm{MnO}_{2}$ (99.99\% purity) by heating in air at $923 \mathrm{~K}$ for $24 \mathrm{~h}$. The starting mixture was placed in $\mathrm{Au}$ capsules and treated at $6 \mathrm{GPa}$ and about $1550 \mathrm{~K}$ for $2 \mathrm{~h}$ (heating time to the synthesis temperature was $10 \mathrm{~min}$ ) in a belt-type high-pressure apparatus. After the heat treatment, 
the samples were quenched to room temperature, and the pressure was slowly released. All the samples obtained were black polycrystalline pellets. X-ray diffraction data were collected using a Rigaku MiniFlex 600 with $\mathrm{Cu} K_{\alpha}$ radiation. dc magnetic susceptibility was measured using a Quantum Design SQUID magnetometer (MPMS-1T) on cooling from 350 to $2 \mathrm{~K}$ in $H=100$ Oe. $M-H$ curves were measured on a MPMS-XL-7T. Specific heat $c_{p}$ was recorded between 2 and $300 \mathrm{~K}$ on cooling and warming by a pulse relaxation method using a Quantum Design PPMS at $H=0 \mathrm{~T}$. Dielectric properties were measured using a NOVOCONTROL Alpha-A High Performance Frequency Analyzer between 3 and $300 \mathrm{~K}$ on cooling and heating, and at $H=0$ and $9 \mathrm{~T}$. Neutron powder diffraction (NPD) measurements were performed on the WISH time-of-flight diffractometer [14] at ISIS, the UK Neutron and Muon Spallation Source, where the sample was loaded into a 3-mm-diameter cylindrical vanadium can and mounted within a ${ }^{4} \mathrm{He}$ cryostat. NPD data were collected with high counting statistics at a fixed temperature within each magnetic phase, and with lower counting statistics on warming in the temperature range 1.5 to $230 \mathrm{~K}$. The crystal and magnetic structures detailed below were refined using FULLPROF [15] against data measured in detector banks at average $2 \theta$ values of $58^{\circ}$ and $154^{\circ}$.

The temperature dependence of the specific heat of DMO [Fig. 1(a)] showed two phase transitions at $T_{C} \simeq$ $95 \mathrm{~K}$ and $T_{\text {Dy }} \simeq 5.5 \mathrm{~K}$. Thermal hysteresis was observed at $T_{C}$, which indicates a first order phase transition. The specific heat peaked at $T_{\mathrm{Dy}}$ is quantitatively consistent with a Schottky anomaly calculated for a two-level system with an energy gap of $\Delta=2 k_{B} T_{\text {Dy }}$ [Fig. 1(a), inset], which is likely related to a splitting of the Dy ground state Kramers doublets, discussed below. Magnetometry data plotted in Fig. 1(b) indicated that both transitions involved magnetic degrees of freedom, with changes below $T_{C}$ being consistent with the onset of a magnetically ordered phase composed of ferromagnetic sublattices.

To establish the microscopic origin of the observed phase transitions, NPD data were collected in the paramagnetic phase above $T_{C}$ [Fig. 2(a)], between $T_{C}$ and $T_{\text {Dy }}$ [Fig. 2(b)], and below $T_{\text {Dy }}$ [Fig. 2(c)]. Every diffraction peak observed in the paramagnetic phase could be accounted for by the $I 2 / m$ crystal structure common to many $R \mathrm{Mn}_{7} \mathrm{O}_{12}$ compounds [16-19], indicating that the sample was single phase (consistent with laboratory based $\mathrm{x}$-ray diffraction measurements [20]). Rietveld refinement of the respective structural parameters (Table I) including $A$-site cation disorder gave an excellent fit to the data, as shown in Fig. 2(a) $\left(R=2.86 \%, w R=2.53 \%, R_{\text {Bragg }}=3.70 \%\right)$. The sample composition was refined to $\left(\mathrm{Dy}_{0.91} \mathrm{Mn}_{0.09}\right) \mathrm{Mn}_{7} \mathrm{O}_{12}$, in perfect agreement with the target composition of the synthesis. Bond valence sum calculations gave a Dy oxidation state of +2.24 , showing that this nominally trivalent cation was considerably underbonded in the paramagnetic phase.

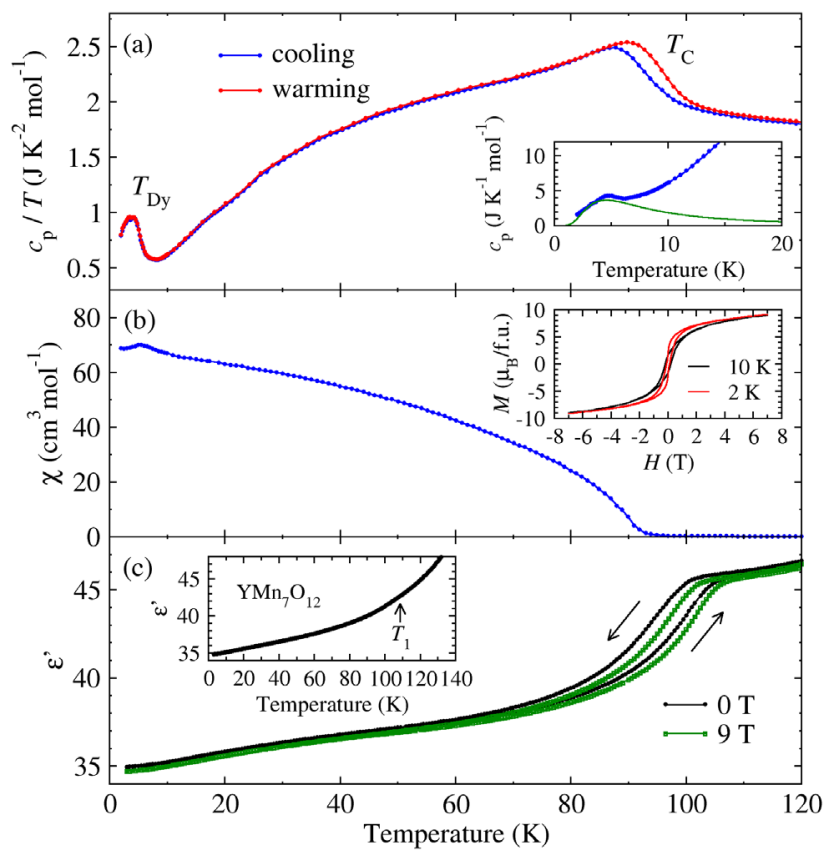

FIG. 1. (a) Specific heat of DMO divided by temperature, measured on cooling (blue) and warming (red). The inset highlights the low-temperature specific heat data (blue), with a calculated Schottky anomaly shown in green. (b) The 100 Oe field cooled, measured on cooling dc magnetic susceptibility of DMO. The inset shows $M-H$ magnetization loops measured above and below $T_{\mathrm{Dy}}$. (c) The real part of the dielectric constant of DMO measured at $665 \mathrm{kHz}$ on cooling and warming (indicated by arrows) in a $0 \mathrm{~T}$ (black) and $9 \mathrm{~T}$ (green) magnetic field. The inset shows the $0 \mathrm{~T}$ dielectric constant measured from $\mathrm{YMn}_{7} \mathrm{O}_{12}$.

On cooling through $T_{C}$ additional diffraction intensities appeared that could be indexed with the $\Gamma$ point, $\mathbf{k}=$ $(0,0,0)$ propagation vector. These intensities were well accounted for by a collinear ferrimagnetic structure composed of all Mn moments, as found below $T_{C}$ in most other $R \mathrm{Mn}_{7} \mathrm{O}_{12}$ compounds [19,21]. Refinement of this magnetic structure against data measured at $20 \mathrm{~K}$ [Fig. 2(b)] gave excellent agreement factors $(R=3.11 \%, w R=3.23 \%$, $\left.R_{\text {mag }}=1.76 \%\right)$. $A$ - and $B$-site $\mathrm{Mn}$ moments refined to $3.18(4)$ and 3.17(2) $\mu_{B}$, respectively, and were found to lie in the $a c$ plane, $33.5(6)^{\circ}$ from the $c$ axis (left-handed rotation about $b$ ). The magnetic structure is illustrated in Fig. 3(a), which shows the relative moment directions between symmetry inequivalent sublattices.

Below $T_{\text {Dy }}$ certain $\Gamma$-point intensities increased, as highlighted in the inset of Fig. 2(c). Structure factor calculations showed that these intensity changes could be accounted for by ferromagnetic ordering of Dy moments parallel to the $b$ axis [Fig. 3(b)], consistent with changes in the field dependent magnetization shown in the inset of Fig. 1(b). This scenario was confirmed by Rietveld refinement against the NPD data measured at $1.5 \mathrm{~K}$ [Fig. 2(c)], giving Dy moments of 3.0(1) $\mu_{B} \| b$. The refinement also gave a global $10(1)^{\circ}$ rotation of the Mn moments out of the 


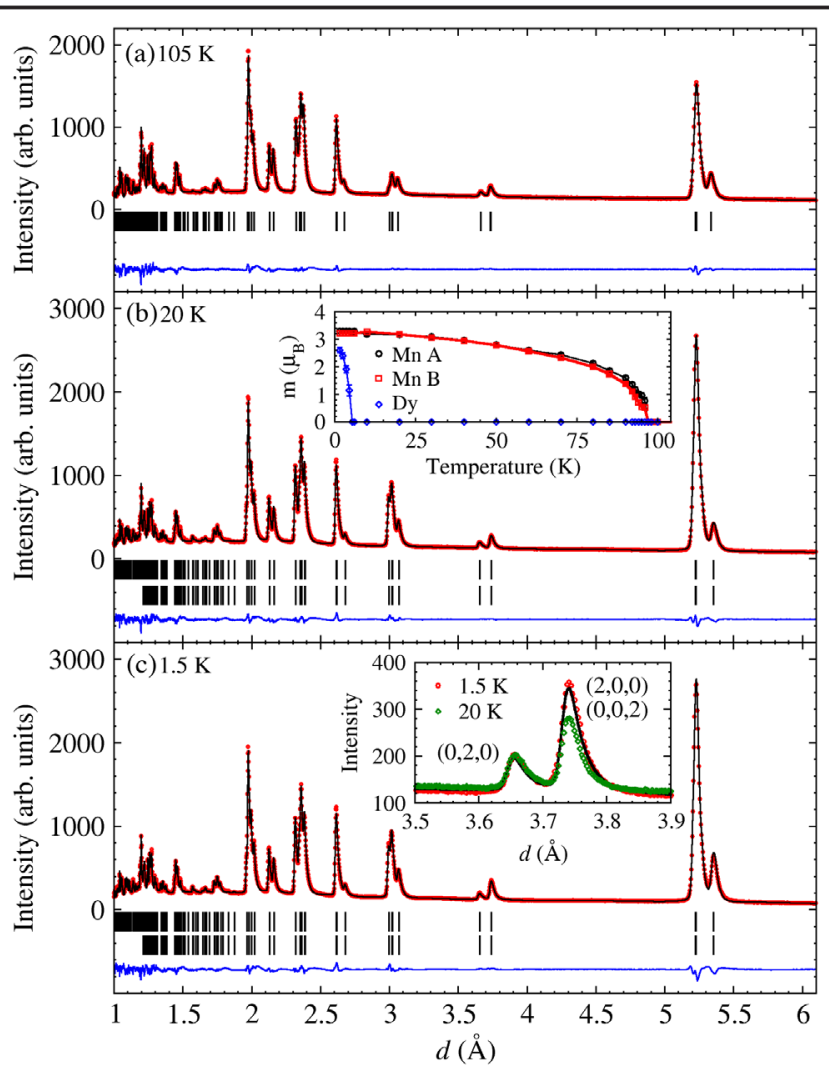

FIG. 2. Neutron powder diffraction data (red circles) measured in (a) the paramagnetic phase above $T_{C}$, (b) between $T_{C}$ and $T_{\text {Dy }}$, and (c) below $T_{\text {Dy }}$. Fits to the data are shown as solid black lines, the differences between data and fit are shown as solid blue lines, and the position of nuclear and magnetic Bragg peaks are marked by top and bottom black tick marks, respectively. The inset of (b) shows the temperature dependence of the Dy, $A$-site $\mathrm{Mn}$, and $B$-site Mn moments, and the inset of (c) highlights diffraction intensity originating in the ferromagnetic ordering of Dy moments parallel to $b$.

ac plane, likely due to $f$ - $d$ exchange coupling, while the in-plane moment direction remained constant, refining to $33(2)^{\circ}$ from the $c$ axis. Mn moment magnitudes were assumed to have saturated just above $T_{\mathrm{Dy}}$, and excellent agreement with the data was achieved $(R=3.17 \%$, $w R=3.11 \%, R_{\mathrm{mag}}=2.8 \%$ ). The ordering of Dy moments below $T_{\text {Dy }}$ was further corroborated by the magnitude of the heat capacity anomaly described above [Fig. 1(a)], which was quantitatively consistent with the splitting of every Dy ground state Kramers doublet.

The crystal and magnetic structures established above were refined against variable temperature NPD data. The evolution of the magnetic moments is shown in the inset of Fig. 2(b), which demonstrates critical behavior at both $T_{C}$ and $T_{\mathrm{Dy}}$. Furthermore, anomalously large steps in the lattice parameters [Figs. 4(b)-4(d)], negative thermal expansion [Fig. 4(e)], and a greater than twofold increase in the Dy atomic displacement parameter $U_{\text {iso }}$ were found to accompany the onset of ferrimagnetic order at $T_{C}$. The (a) $20 \mathrm{~K}$
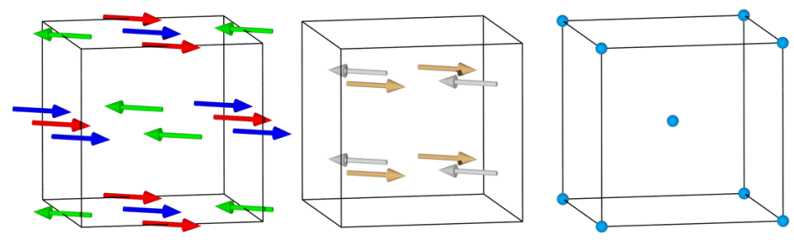

(b) $1.5 \mathrm{~K}$
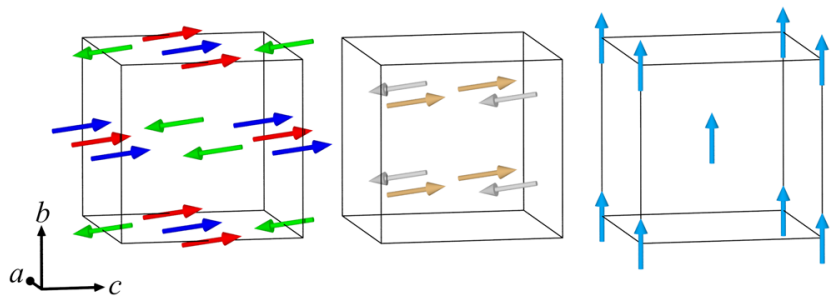

FIG. 3. The magnetic structure of DMO (a) between $T_{C}$ and $T_{\text {Dy }}$ and (b) below $T_{\text {Dy }}$. Mn1, Mn2, and Mn3 $A$-site moments are drawn to the left in blue, green, and red, respectively. Mn4 and Mn5 $B$-site moments are drawn in the center in gold and silver, respectively. The Dy sites are drawn to the right, colored blue. The $I 2 / m$ unit cell is drawn as thin black lines.

$U_{\text {iso }}$ parameters of Dy, $A$-site $\mathrm{Mn}, B$-site $\mathrm{Mn}$, and O were refined independently and found to be uncorrelated; hence the anomalous increase of Dy $U_{\text {iso }}$ specifically indicated the spontaneous development of off-centering displacements of Dy (electric dipoles) below $T_{C}$. Refinement of possible ferroelectric crystal structures (polar space groups Im and I2 [22]) against NPD data measured at $20 \mathrm{~K}$ gave Dy displacements smaller than their standard error, and further increased $U_{\text {iso }}$. An antiferroelectric crystal structure would give rise to well-isolated, superstructure diffraction intensities, which were not observed in the data. We therefore ruled out long-range ordering of electric dipoles, and instead considered the development of non-symmetrybreaking, statistically disordered Dy displacements averaged over 4 symmetry equivalent sites, i.e., a spontaneous

TABLE I. DMO crystal structure parameters refined at $105 \mathrm{~K}$ $[I 2 / m, a=7.4538(1) \AA, b=7.31225(8) \AA, c=7.4577(1) \AA$, and $\beta=91.206(1)^{\circ}$ ].

\begin{tabular}{lcccc}
\hline \hline Atom & $x$ & $y$ & $z$ & $U_{\text {iso }}\left(\AA^{2}\right)$ \\
\hline Dy(Mn) & 0 & 0 & 0 & $0.002(1)$ \\
Mn1 & 0 & $1 / 2$ & 0 & $0.0071(9)$ \\
Mn2 & $1 / 2$ & 0 & 0 & $0.0071(9)$ \\
Mn3 & $1 / 2$ & $1 / 2$ & 0 & $0.0071(9)$ \\
Mn4 & $1 / 4$ & $1 / 4$ & $1 / 4$ & $0.0027(8)$ \\
Mn5 & $1 / 4$ & $1 / 4$ & $3 / 4$ & $0.0027(8)$ \\
O1 & $0.1665(5)$ & 0 & $0.3015(5)$ & $0.011(1)$ \\
O2 & $0.1803(7)$ & 0 & $0.6841(7)$ & $0.012(1)$ \\
O3 & $0.0132(4)$ & $0.3081(4)$ & $0.1723(4)$ & $0.013(1)$ \\
O4 & $0.3123(4)$ & $0.1749(4)$ & $-0.0121(5)$ & $0.014(1)$ \\
\hline \hline
\end{tabular}



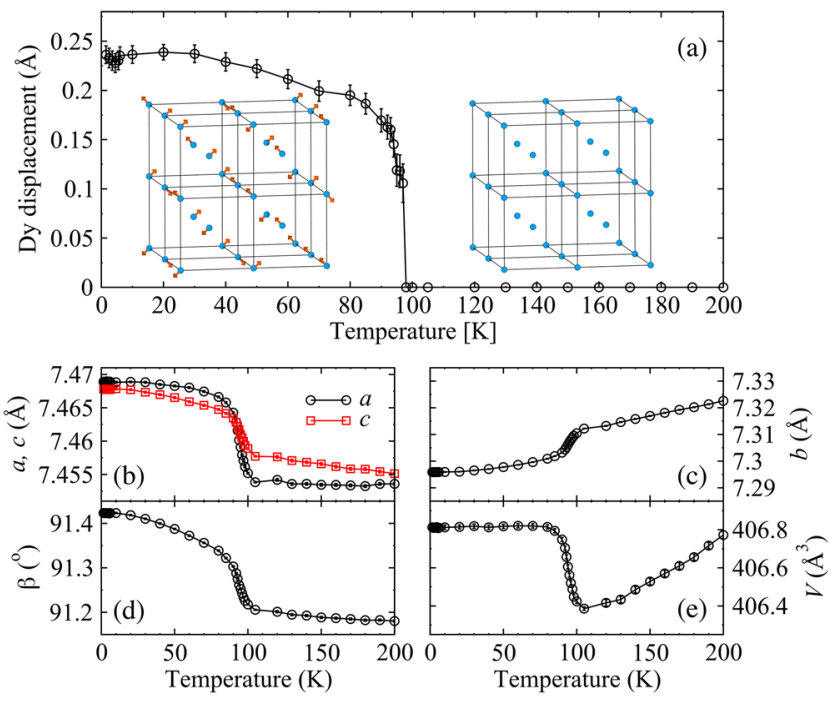

FIG. 4. (a) Dy displacement magnitude, (b)-(d) lattice parameters, and (e) unit cell volume, refined as a function of temperature. The insets of (a) illustrate the formation of a Dy dipolar glass.

dipolar glass [Fig. 4(a), inset]. The variable temperature refinements were repeated, and a disordered Dy displacement was found to develop with critical behavior below $T_{C}$ [Fig. 4(a)], while maintaining typical values of $U_{\text {iso }}$. We note that the formation of a dipolar glass is consistent with the observed negative thermal expansion, as the Dy ions effectively increase their ionic radii in the disordered state. Furthermore, the Dy bond valence sum increased to +2.33 at $20 \mathrm{~K}$, indicating a significant improvement in its bonding. The emergence of a state of disordered electric dipoles below a classical phase transition is extremely rare, if not unique. To the best of our knowledge, phase transitions known to be associated with an instability toward the formation of electric dipoles always result in well-correlated dipolar ordering.

The apparent magnetostructural phase transition at $T_{C}$ is exemplified by anomalous behavior of the real part of the dielectric constant [Fig. 1(c)]. Frequency dependent measurements [20] demonstrated that data measured at $665 \mathrm{kHz}$ revealed the intrinsic dielectric behavior of DMO. On cooling through $T_{C}$ there occurred an $\sim 20 \%$ drop in the powder-averaged dielectric constant, reminiscent of that observed in organic or inorganic hybrid perovskites due to the "freezing-out" of $A$-site dipolar degrees of freedom [23]. No dielectric anomaly was observed at the purely magnetic phase transition of $\mathrm{YMn}_{7} \mathrm{O}_{12}$ [21] [Fig. 1(c), inset], which demonstrates that the anomaly is associated with the Dy dipolar glass. The dielectric constant showed thermal hysteresis at $T_{C}$, in good agreement with the heat capacity data, and again consistent with a first order phase transition. Furthermore, the dielectric anomaly can be tuned by an applied magnetic field [Fig. 1(c)], which demonstrated significant magnetostructural coupling at $T_{C}$.
No anomalous features at $T_{C}$ were observed in the imaginary part of the dielectric constant [20], indicating that the dielectric behavior observed at $T_{C}$ is not simply related to a change in conductivity.

Coupling between ordered magnetic and disordered electric dipoles in strongly correlated electron systems is unusual, yet here it can be described using a conventional phenomenological theory. The dipolar glass does not break any macroscopic symmetry; hence the corresponding order parameter $\delta$ is invariant under all symmetry operations of the paramagnetic space group and transforms as the totally symmetric irreducible representation (irrep) $\Gamma_{1}^{+}$. The reducible $\Gamma$-point magnetic representation for the $\mathrm{Mn}$ and $\mathrm{Dy}$ Wyckoff positions decomposes into two, 1D irreps, $m \Gamma_{1}^{+}$ and $m \Gamma_{2}^{+}$[24]. The ferrimagnetic order parameter $\eta$ transforms by the $m \Gamma_{2}^{+}$irrep (magnetic space group $I 2^{\prime} / \mathrm{m}^{\prime}$ ). Given that $m \Gamma_{2}^{+}$is $1 \mathrm{D}, \eta^{2}$ must also be invariant under all symmetry operations of the paramagnetic group. Thus, the linear-quadratic free-energy invariant $\delta \eta^{2}$ is allowed by symmetry and represents the coupling between ferrimagnetism and the dipolar glass. At the microscopic level this coupling is likely mediated by strain, as the magnetic superexchange interactions are known to be very sensitive to strain dependent structural parameters such as interatomic distances and bond angles [25]. The general Landau-type phenomenological theory that includes linear-quadratic coupling between order parameters with distinct instabilities indicates that in some circumstances a single first order phase transition is possible when condensation of one order parameter triggers the onset of another [26,27]. The magnetostructural phase transition in DMO is a clear realization of this scenario. The coupling implies that the non-symmetry-breaking strain acts as a conjugate field for both structural and magnetic instabilities, making this system attractive for thin film studies where the strains can be tuned via choice of substrate.

In DMO, the spontaneous formation of polar displacements of underbonded Dy may be driven by the quasi-JahnTeller effect [5]. To the best of our knowledge, in perovskite-derived materials this effect has only been observed for $B$-site cations, as the bonding requirements of $A$-site cations are usually met by octahedral tilts and rotations [11]. However, in the quadruple perovskites specific octahedral tilts are required to establish a square planar coordination for the majority $(\mathrm{Mn}) A$-site cations, leaving little flexibility for the bonding of the minority $(R)$ $A$-site species. In this case, one might expect quasi-JahnTeller displacements of underbonded $R$ cations (those with a relatively small ionic radii), in analogy with polar displacements usually found at perovskite $B$ sites $[5,6]$. Indeed, similar structural behavior to that described above has been observed in $\mathrm{YMn}_{7} \mathrm{O}_{12}$, which undergoes a purely structural phase transition at $200 \mathrm{~K}$, independent of lower-temperature ferrimagnetic order [21]. This transition also involved a large monoclinic strain, negative thermal 
expansion, and off-centering displacements of yttrium, which, together with an ordered pattern of oxygen displacements, improved the bonding conditions of underbonded yttrium [21,28,29]. A NPD study [21] found no evidence for the ordering of yttrium polar displacements, whereas a later single crystal x-ray diffraction study [29] reported partial antiferroelectric order.

The dipolar instabilities found in both $\mathrm{Dy}_{1-\delta} \mathrm{Mn}_{7+\delta} \mathrm{O}_{12}$ and $\mathrm{YMn}_{7} \mathrm{O}_{12}$ serve as proof of principle for the materials design strategy proposed in the Introduction, and importantly, the results on $\mathrm{Dy}_{1-\delta} \mathrm{Mn}_{7+\delta} \mathrm{O}_{12}$ demonstrate that cross-coupling between magnetic and electric dipoles can be achieved. Many different compositions of quadruple perovskite $R A_{3}^{\prime} B_{4} \mathrm{O}_{12}$ have been reported [30], and the recently discovered $A$-site columnar ordered quadruple perovskites $R_{2} A^{\prime} A^{\prime \prime} B_{4} \mathrm{O}_{12}$ [31] are also gaining considerable attention. In both compositionally rich material families, $A$-site cation ordering and octahedral tilting are mutually tied to meet the criteria of structural stability, making them potential hosts of $A$-site polar instabilities, established via the quasi-Jahn-Teller effect, and coupled to magnetic degrees of freedom.

R. D. J. acknowledges support from a Royal Society University Research Fellowship. K. Y. and A. A. B. acknowledge JSPS KAKENHI Grant No. JP20H05276, a research grant (40-37) from Nippon Sheet Glass Foundation for Materials Science and Engineering, and Innovative Science and Technology Initiative for Security (Grant No. JPJ004596) from Acquisition, Technology, and Logistics Agency (ATLA), Japan.

*roger.johnson@ucl.ac.uk

[1] J. F. Scott, Science 315, 954 (2007).

[2] R. A. Cowley, S. N. Gvasaliya, S. G. Lushnikov, B. Roessli, and G. M. Rotaru, Adv. Phys. 60, 229 (2011).

[3] X. Hao, J. Zhai, L. B. Kong, and Z. Xu, Prog. Mater. Sci. 63, 1 (2014).

[4] N. A. Spaldin and R. Ramesh, Nat. Mater. 18, 203 (2019).

[5] I. B. Bersuker, Chem. Rev. 113, 1351 (2013).

[6] M. Acosta, N. Novak, V. Rojas, S. Patel, R. Vaish, J. Koruza, G. A. Rossetti, and J. Rdel, Appl. Phys. Rev. 4, 041305 (2017).

[7] C. Michel, J.-M. Moreau, G. D. Achenbach, R. Gerson, and W. James, Solid State Commun. 7, 701 (1969).

[8] F. Mezzadri, G. Calestani, M. Calicchio, E. Gilioli, F. Bolzoni, R. Cabassi, M. Marezio, and A. Migliori, Phys. Rev. B 79, 100106(R) (2009).

[9] A. K. Tagantsev, K. Vaideeswaran, S. B. Vakhrushev, A. V. Filimonov, R. G. Burkovsky, A. Shaganov, D. Andronikova, A. I. Rudskoy, A. Q. R. Baron, H. Uchiyama, D. Chernyshov, A. Bosak, Z. Ujma, K. Roleder, A. Majchrowski, J.-H. Ko, and N. Setter, Nat. Commun. 4, 2229 (2013).
[10] N. A. Benedek and C. J. Fennie, Phys. Rev. Lett. 106, 107204 (2011).

[11] P. M. Woodward, Acta Crystallogr. Sect. B 53, 44 (1997).

[12] S. Geller, J. Chem. Phys. 24, 1236 (1956).

[13] H. Okamoto, M. Karppinen, H. Yamauchi, and H. Fjellvg, Solid State Sci. 11, 1211 (2009).

[14] L. C. Chapon, P. Manuel, P. G. Radaelli, C. Benson, L. Perrott, S. Ansell, N. J. Rhodes, D. Raspino, D. Duxbury, E. Spill, and J. Norris, Neutron News 22, 22 (2011).

[15] J. Rodríguez-Carvajal, Physica (Amsterdam) 192B, 55 (1993).

[16] B. Bochu, J. Chenavas, J. Joubert, and M. Marezio, J. Solid State Chem. 11, 88 (1974).

[17] A. Prodi, E. Gilioli, R. Cabassi, F. Bolzoni, F. Licci, Q. Huang, J. W. Lynn, M. Affronte, A. Gauzzi, and M. Marezio, Phys. Rev. B 79, 085105 (2009).

[18] F. Mezzadri, M. Calicchio, E. Gilioli, R. Cabassi, F. Bolzoni, G. Calestani, and F. Bissoli, Phys. Rev. B 79, 014420 (2009).

[19] R. D. Johnson, D. D. Khalyavin, P. Manuel, L. Zhang, K. Yamaura, and A. A. Belik, Phys. Rev. B 98, 104423 (2018).

[20] See Supplemental Material at http://link.aps.org/ supplemental/10.1103/PhysRevLett.125.097601 for laboratory based $\mathrm{x}$-ray diffraction data and temperature dependencies of the complex dielectric constant measured at different frequencies.

[21] R. D. Johnson, D. D. Khalyavin, P. Manuel, Y. Katsuya, M. Tanaka, Y. Matsushita, L. Zhang, K. Yamaura, and A. A. Belik, Phys. Rev. B 99, 024107 (2019).

[22] The lower $P 1$ symmetry is reached through a linear combination of modes associated with the $I 2$ and $I m$ space groups. Hence, if $I 2$ and $I m$ are independently ruled out, so too is $P 1$.

[23] W. Zhang, Y. Cai, R.-G. Xiong, H. Yoshikawa, and K. Awaga, Angew. Chem. Int. Ed. 49, 6608 (2010).

[24] B. J. Campbell, H. T. Stokes, D. E. Tanner, and D. M. Hatch, J. Appl. Crystallogr. 39, 607 (2006).

[25] J. B. Goodenough, Magnetism and the Chemical Bond (John Wiley and Sons, New York, 1963).

[26] P. Toledano and V. Dmitriev, Reconstructive Phase Transitions: In Crystals and Quasicrystals (World Scientific, Singapore, 1996).

[27] E. K. H. Salje and M. A. Carpenter, J. Phys. Condens. Matter 23, 462202 (2011).

[28] M. Verseils, F. Mezzadri, D. Delmonte, B. Baptiste, Y. Klein, S. Shcheka, L. C. Chapon, T. Hansen, E. Gilioli, and A. Gauzzi, Phys. Rev. Mater. 1, 064407 (2017).

[29] M. Verseils, F. Mezzadri, D. Delmonte, R. Cabassi, B. Baptiste, Y. Klein, G. Calestani, F. Bolzoni, E. Gilioli, and A. Gauzzi, Inorg. Chem. 58, 14204 (2019).

[30] A. N. Vasilev and O. S. Volkova, Low Temp. Phys. 33, 895 (2007).

[31] A. A. Belik, D. D. Khalyavin, L. Zhang, Y. Matsushita, Y. Katsuya, M. Tanaka, R. D. Johnson, and K. Yamaura, Chem. Phys. Chem. 19, 2449 (2018). 